\title{
Cultura económica de los microproductores a través de la vinculación universitaria: Una experiencia ecuatoriana
}

Rosa González Zambrano' Escuela Superior Politécnica de Manabí

rgonzales@espam.edu.ec

https://orcid.org/0000-0003-0960-1817

Benjamín Wilson León Valle ${ }^{2}$ Universidad Estatal Península de Santa Elena bleon@upse.edu.ec https://orcid.org/0000-0002-3374-5207

René Garzozi Pincay ${ }^{3}$ Universidad Estatal Península de Santa Elena rgarzozi@upse.edu.ec https://orcid.org/0000-0003-1779-9384

Lourdes Crespo Zafra ${ }^{4}$

Universidad de Camagüey lourdes.crespo@reduc.edu.cu https://orcid.org/0000-0002-4799-3447

Jorge Saavedra Palma ${ }^{5}$ Universidad Estatal Península de Santa Elena jsaavedra@upse.edu.ec https://orcid.org/0000-0001-5722-7861 


\title{
Resumen
}

El objetivo de este trabajo es identificar limitaciones en la orientación educativa-social del proceso de vinculación de la universidad con el desarrollo de una cultura económica en microproductores. Se presenta una estrategia de extensión universitaria manifestando procesos característicos de educación popular con particularidades de los sujetos y contexto actuación. La estrategia es resultado de gestión de métodos y técnicas de investigación pedagógica concluyendo que deben desarrollarse desde la responsabilidad social universitaria, para que los microproductores puedan desarrollarse.

Palabras clave: proceso, microproductores, vinculación, universidad.

\begin{abstract}
The objective of this work is to identify limitations in the educational-social orientation of the process of linking the university with the development of an economic culture in micro-producers. A university extension strategy is presented, showing characteristic processes of popular education with particularities of the subjects and context of action. The strategy is the result of managing pedagogical research methods and techniques, concluding that they must be development from university social responsibility, so that micro-producers can develop.
\end{abstract}

Keywords: process, microproductors, linkage, university. 


\section{Introducción}

La universidad es una institución que aparece como respuesta a las necesidades de la sociedad, el carácter histórico de su surgimiento y desarrollo ha propiciado la aparición de sus procesos sustantivos.

En las últimas décadas ha crecido la demanda social a la universidad, a sus procesos, al vínculo con el entorno socio productivo y cultural, una mayor correspondencia con la práctica para el logro de los objetivos de la formación del profesional, la necesidad de ir desde el desarrollo de los procesos universitarios a la búsqueda de soluciones a los problemas sociales de las regiones donde actúan. Esta necesidad de satisfacción de demandas económicas, como del desarrollo social y cultural, lleva a las universidades a responder con un proceso dinamizador que propicia su liderazgo como institución social (Robalino, 2005).

Las universidades han realizado diversas innovaciones curriculares para incorporar el desarrollo del espíritu emprendedor en todas sus carreras, no solamente aquellas vinculadas al área de los negocios. También se han implementado programas complementarios de vinculación con la sociedad y el mundo del trabajo y la producción, incorporación de los jóvenes en asesoría a microempresas, interrelación entre los distintos niveles de la educación superior, variedad de programas y de calificaciones de competencias, entre los más relevantes (Garzozi, 2014).

Aunque en varios trabajos se aborda la problemática del desarrollo de los procesos sustantivos de la educación superior y particularmente el posgrado en estrecha relación con el desarrollo regional (Garzón, 2017), es en este contexto donde el proceso de vinculación (extensión universitaria) que aunque recibe influencias de los otros procesos, aún no logra un elevado impacto en el accionar de las universidades para garantizar su encargo social, económico, ambiental y cultural en la nueva universidad ecuatoriana, si bien el artículo 351 de la Constitución de la República del Ecuador indica que: "El sistema de educación superior estará articulado al sistema nacional de educación y al Plan Nacional de Desarrollo", (Constitución de la República del Ecuador, 2008).

En este contexto los procesos educacionales alcanzan trascendencia e implicaciones. En lo específico la Educación Superior se convierte en un escenario que aglutina y a la vez irradia múltiples facetas del desarrollo humano y social (Barnett, 2002; Boni, 2006; De Sousa, 2010); una de las cuales es la "económica" que, como proceso vital, dinamiza la existencia humana y está presente en casi todos los móviles de actuación de ser humano; por lo que, el logro de una cultura de ese tipo influye acentuadamente en la formación de la personalidad, la cual, cualquier sociedad, como lo es la ecuatoriana, anhela correspondencia con su sistema social. De ahí que el tema de esta investigación revele una pertinencia social e importancia desde una perspectiva educativa a partir de los fundamentos aportados por Achugar (2000), Cabrera (2017), Araujo (2009) (BID, 2009).

Referido a la cultura económica, como primer acercamiento, se valora que el tema ha sido poco trabajado desde referentes educativos; no obstante, se pueden leer algunas ideas sugestivas que recalcan la importancia de materializarla desde la asimilación dialéctica del pensamiento económico universal contemporáneo, integrándolo con el nacional y con la realidad histórico- 
concreta particular; visto así, esta debe pasar por la elevación de la conciencia crítica de la ciudadanía desde la educación, en específico, desde la universidad como motor de crecimiento económico, tecnológico y empresarial, pero también, como un factor de creación (Alberto Peña y Pablo Guerra, 1990; Castells, 2004; Sabalain, 2011; FAO-FIDA-OIT, 2010).

En la actualidad, en el Ecuador, el incremento de microproductores se ha caracterizado por una gran diversidad de iniciativas realizadas por una multiplicidad de actores. En este contexto la demanda por servicios de apoyo y desarrollo, crece, lo que evidencia la importancia que estos tienen en la economía del país; sin embargo, estos actores no se encuentran capacitados a la hora de asumir un rol protagónico, esto es, al margen de que los programas institucionales-estatales tienen problemas en su concreción.

Esa situación afecta a todos los responsables de su materialización; por lo que es importante generar alternativas educativas desde la Universidad que favorezcan la formación de una cultura económica.

Los territorios entregan los problemas del desarrollo regional a resolver por las universidades, mediante la investigación y la formación académica. A partir de estos problemas, se puede proceder a ver las posibilidades reales de la Escuela Superior Politécnica Agropecuaria de Manabí, Manuel Félix López (ESPAM MFL) para la solución de los mismos, constatándose que en la región existe un potencial para ser desarrollado, por tanto, es en la preparación de la gente, del entorno, que se logrará el perfeccionamiento de la educación, en aras de encontrar la solución a los dilemas y problemas sociales de la región (Garzón, 2017; Núñez, 2014), citando el informe de la Comisión Mundial de Cultura y Desarrollo, afirma que: El desarrollo en el Siglo XXI será cultural o no será (...). Las respuestas han de traducirse en transformaciones educativas, donde las universidades han de erigirse en gestoras fundamentales del desarrollo social y con ello rescatar su misión de ser la conciencia cultural de la sociedad, no sólo en su concepción general, sino en sus enfoques pedagógicos y en los contenidos de los procesos formativos (...), (p. 2).

En ese estado de cosas es que surgen corrientes ideológicas y políticas que se vinculan directamente a múltiples problemas económicos; pero, a la vez, no solucionan o agudizan la pobreza y el subdesarrollo revelando la actualidad de cualquier investigación donde se manifiesten las relaciones universidad- sociedad. La (UNESCO, 2009), reconoce que: “(...) los sistemas de educación superior deberían aumentar su capacidad para (...) transformarse y provocar el cambio, para atender las necesidades sociales y fomentar la solidaridad y la igualdad..." (p. 3).

A partir de la experiencia empírica de los autores de la investigación han identificado las contradicciones preponderantes:

- La extensión aún desarrolla procesos focalizados en el ámbito artístico y deportivo y no logra en su magnitud un vínculo universidad-sociedad, que contribuya al desarrollo socioeconómico del contexto.

- No existen acciones educativas manifiestas o proyectos, desde la extensión, para lograr una cultura económica en los microproductores agrícolas. 
- Aumenta el número de microproductores que no logran avances productivos y/o pierden sus bienes por la ausencia de una educación encaminada a concientizarlos sobre el alcance de sus decisiones y acciones en tal sentido.

De acuerdo con las situaciones detectadas y las demandas sociales para el logro del propósito de la investigación se evidencia la contradicción existente entre las exigencias del vínculo universidad-sociedad y la necesidad de desarrollar una cultura económica de los microproductores.

El rol que cumplen las universidades ante los procesos de cambio de la sociedad hace de estas instituciones sociales importantes centros de cambios, capaces de dar respuestas a los crecientes retos y desafíos provenientes de una economía insertada en un proceso de globalización que genera grandes problemas sociales en la mayor parte del mundo.

El objetivo se dirige a presentar una estrategia de extensión universitaria donde se pongan de manifiesto procesos característicos de la educación popular a tono con las características de los sujetos y el contexto de actuación.

\section{Metodología}

Para la realización de la investigación se empleó los siguientes métodos y técnicas: la modelación, para la representación de los nexos y las relaciones de procesos vinculados al desarrollo de una cultura económica de los microproductores; la observación que posibilitó constatar los elementos que se ponen de manifiesto en la actuación de los microproductores y permitió su caracterización en la Universidad; Talleres (con docentes y estudiantes de la ESPAM MFL, microproductores agrícolas del cantón Bolívar, miembros de la dirección universitaria y oficiales de crédito de las instituciones financieras) que permitieron examinar el estado actual de la problemática; análisis documental: para obtener información sobre el estado actual del problema contribuyendo a sistematizar enfoques y concepciones.

La introducción parcial de la estrategia comenzó en el año 2016 con un preexperimento, en el cantón Bolívar de la provincia Manabí y se extendió hasta mediados del año 2017. Participaron directamente cinco microproductores acompañados de los familiares que vivían con ellos, un total de 15 estudiantes de la ESPAM-MFL, seis profesores de dicho centro y 5 investigadores.

\section{Argumentación de la Estrategia Educativa de Extensión Universitaria para el desarrollo de una cultura económica en los microproductores agrícolas}

Las estrategias requieren delimitar problemas, proponer objetivos a alcanzar, programar recursos y planificar acciones que den respuesta al problema que se necesita solucionar. Estas se van a caracterizar por su flexibilidad y por propiciar la posibilidad de ser modificadas en correspondencia con las necesidades y cambios que se operen en los que en ellas participan. Las estrategias deben desarrollar en la enseñanza y la formación el aprendizaje basado en problemas (ABP), evidenciando las contradicciones del proceso y la necesidad de un constante perfeccionamiento del mismo. 
Los autores se adscriben a la concepción de quienes reflexionan que una estrategia constituye el conjunto de influencias y acciones que permiten la formación, con el fin de que los estudiantes impulsen su desarrollo. Se tiene en cuenta, además las diferentes dimensiones que deben cumplir: innovadora, prospectiva y orientadora.

Se toman igualmente los procesos declarados de la educación popular propuestos por Freire (1975-1976) y sus seguidores tales como De la Torre (2001), Geerlings (1999), Antillón (2002), Van de Velde (2002), Gómez (2001), Russo (2000), Sojo (2007), Barretto (2007), Jara (2010), Acconcia (2017), los cuales denotan lo siguiente: experiencia, concienciación-reflexión, participación criticoreflexiva, comunicación dialógica, integración transformación y contextualización.

De los mismos autores citados, su coincidencia sobre la necesidad de que este tipo de educación parta de la realidad de los sujetos desde su condición histórica y de la toma de conciencia crítica en torno a ella; que se utilicen diversas técnicas participativas que generen cooperación, solidaridad y corresponsabilidad; que valúen la cultura popular y la identidad cultural propia; que asuman los modos de conocer y permitan adquirir conocimientos propios de las comunidades con las cuales se interactúa; que tomen la realidad como punto de partida para volver a ella y lograr transformarla; que se organice el aprendizaje con la participación de la comunidad; y que se promueva la dialogicidad, la autoevaluación y la autogestión del proceso de formación.

Considerando la conceptualización tomamos la Estrategia Educativa que se sustenta en el modelo educativo de Extensión Universitaria para el desarrollo de una cultura económica en los microproductores agrícolas, desarrollado por (González, 2018).

Esta estrategia debe cumplir con las siguientes funciones:

- Instructiva: en la preparación económica del microproductor

- Educativa: el microproductor participa colaborativa y solidariamente con el agente extensionista y en grupo, realizándose acciones a través de la detección y solución de problemáticas relacionados con la vida laboral y personal que de hecho se constituyen en necesidades sociales de la comunidad.

- Formadora: a partir de acciones insertadas en la estrategia se va dirigiendo el proceso encaminado a la formación de un modo de actuación que sea sostenible.

- Transformadora: a partir de las acciones a realizar por el microproductor se van logrando los conocimientos, habilidades y la educación en valores que le permiten ir logrando una evolución personal y con ello, del entorno en el cual desarrolla su vida.

- Ética: las acciones a realizar están dirigidas a la formación de una conducta ética en cuyo centre estén las relaciones entre los seres humanos-naturaleza-sociedad.

- Política-Ideológica: formar ideas y valores que respondan al desarrollo de una cultura económica de manera comprometida. 
La estructura general de la Estrategia se dimensiona a partir de la organización ofrecida en el subsistema gestión transformadora extensionista para el desarrollo de la cultura económica (González, 2018), donde la extensión se visualiza como un proceso sustantivo, y su dimensión transformadora social como extensión universitaria en sí. La estrategia consta de dos fases en las que aparecen varias etapas. La primera fase: Preparación del sistema universidad-sociedad, consta de cuatro etapas (ver Figura 1).

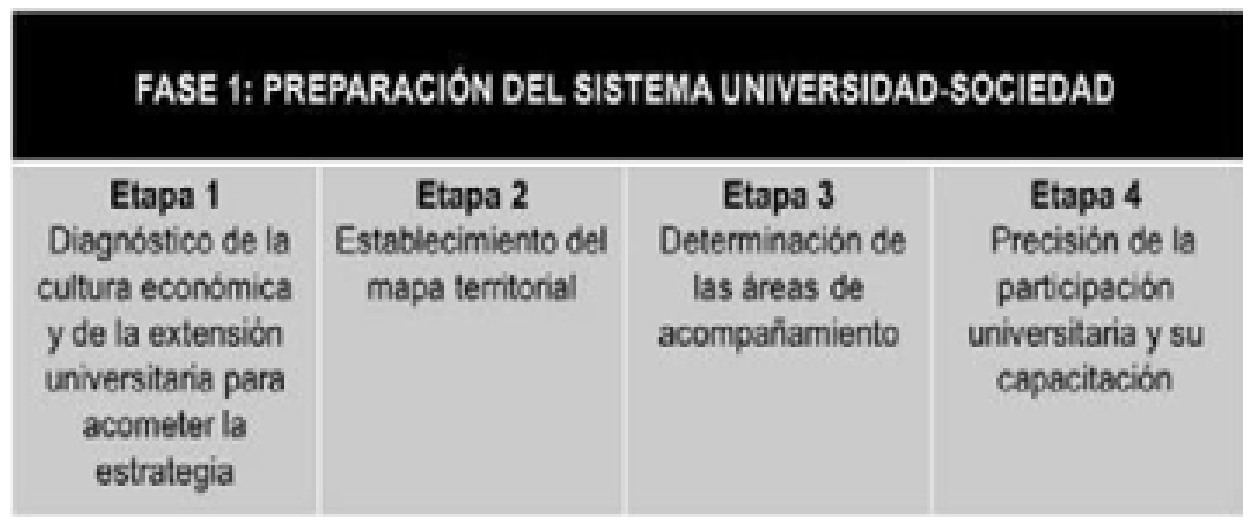

Figura 1. Fase I Preparación del sistema universidad-sociedad.

Fuente: elaboración propia

La segunda fase: Gestión de la instrumentación de la estrategia educativa de extensión universitaria, compuesta por cinco etapas (ver Figura 2).

\begin{tabular}{|c|c|c|c|c|}
\hline \multicolumn{5}{|c|}{$\begin{array}{l}\text { FASE 2: GESTIÓN DE LA INSTRUMENTACIÓN DE LA ESTRATEGIA EDUCATIVA } \\
\text { DE EXTENSIÓN UNIVERSITARIA }\end{array}$} \\
\hline $\begin{array}{c}\text { Etapa } 1 \\
\text { Definición } \\
\text { colectiva de la } \\
\text { propuesta de } \\
\text { formación }\end{array}$ & $\begin{array}{c}\text { Etapa } 2 \\
\text { Determinación } \\
\text { de la } \\
\text { experiencia con } \\
\text { significado y } \\
\text { relevancia }\end{array}$ & $\begin{array}{l}\text { Etapa } 3 \\
\text { Procesos de } \\
\text { reflexión } \\
\text { critica }\end{array}$ & $\begin{array}{c}\text { Etapa } 4 \\
\text { Profundización } \\
\text { temática. }\end{array}$ & $\begin{array}{c}\text { Etapa } 5 \\
\text { Evaluar, } \\
\text { sistematizar e } \\
\text { identificar } \\
\text { nuevas } \\
\text { necesidades }\end{array}$ \\
\hline
\end{tabular}

Figura 2. Fase 2. Gestión de la instrumentación de la estrategia educativa de extensión universitaria

Fuente: elaboración propia

\section{Aplicación de la Estrategia Educativa de Extensión Universitaria con los microproductores agrícolas desde la ESPAM MFL}

Para esto fue necesario preparar a los estudiantes y profesores intervinientes, junto con directivos de investigaciones, posgrado y vinculación, a los que se les explicó la esencia de la Estrategia y se proveyeron de procedimientos participativos para concretar la experiencia. Se comentan algunos resultados de la implementación de la fase 1. 
Sobre el primer aspecto, de esta fase: Preparación del sistema universidad-sociedad, la labor comenzó con el diagnóstico del contexto, que se efectuó a través de una comparación de la cultura económica del microproductor y de la producción del contexto, donde se contrastaron sus prácticas individuales y las de mejor experiencia del cantón (efectividad), de forma tal que los microproductores comprendieran la necesidad de superarse en lo referido al tema económico y finalmente poder transformar sus prácticas.

La primera barrera encontrada en la investigación fue el recelo de algunos de ellos sobre la intervención universitaria y fue necesario impartirles charlas donde se pusieron ejemplos de cómo esta podía ayudarlos y favorecerlos para llegar a transformar sus modos de pensar y actuar en lo referido a la problemática objeto de atención.

Se realizó, además, un diagnóstico de las necesidades individuales de aprendizaje del microproductor y las de la localidad, por lo que se introdujeron en la preparación ya mencionada, algunas charlas e intercambios para que comprendieran que la solución de sus necesidades era aquella que a su vez resolviera las necesidades del contexto rural donde viven.

En este momento también se estableció el mapa territorial, enfocado en un estudio del contexto para conocer e identificar los actores sociales, las organizaciones que allí conviven, las lógicas de circulación de cada actor social y sus relaciones, como escenario de la comunicación, con sus conflictos, sus redes, potencialidades y de recursos, además que ello posibilitó fijar la muestra de microproductores, su localización y tipos de cultivos.

Se llegó a la decisión de tomar el cantón Bolívar; y dentro de él, la comunidad San Miguel de Sarampión, compuesta por 146 familias y 700 habitantes, lugar que es un espacio donde la principal preocupación es la conservación del medio y de los recursos naturales. Se caracteriza por el cultivo orgánico, la reutilización del agua, el uso de baños ecológicos y el reciclaje. Su principal producto es el cacao fino de aroma y los cultivos de ciclo corto, como maíz, arroz, plátano, yuca, camote y cítricos, pero su producción no es tecnificada, el suelo está desgastado por la deforestación, lo que ha causado un empobrecimiento de los suelos y la necesidad de reutilización de las semillas, bajando el nivel de la producción y la rentabilidad, lo que causa el aumento de las tasas de desempleo y emigración.

El cantón presenta una topografía plana en la parte baja por los valles e irregular ondulada y quebrada en la parte alta y montañosa. La altitud es de $21 \mathrm{msnm}$ la parte baja y de $400 \mathrm{msnm}$ en la parte alta. El área montañosa se caracteriza por haber

constituido una reserva natural de bosques de protección, que ha sido degradado por la explotación de la madera y la ampliación de la frontera de tierras para la crianza de ganado vacuno y porcino, en detrimento de los equilibrios ambientales debido a los fuertes impactos negativos que se derivan de la pérdida de la masa boscosa.

También, se precisaron las áreas de acompañamiento por parte de los gestores extensionistas. Dado el resultado del establecimiento del mapa territorial se seleccionan los 5 microproductores con su familia, hijos y familiares en edad escolar que viven con ellos como casos de 
experimentación, a los cuales se le asignó, a cada uno un gestor extensionista (en este caso, estudiantes que, junto a un maestro de la escuela primaria, formaron el grupo de acompañamiento.

De igual manera, se propició la participación de un representante del Gobierno del cantón Bolívar y de las empresas agrícolas, representantes de instituciones financieras del territorio (Cooperativa de Ahorro y Crédito, Banco del Pichincha, Ban-Ecuador); un profesor de la carrera de Administración y otro del área de vinculación con la comunidad.

Estas instituciones han sido reconocidas por los habitantes, puesto que no sólo otorgan créditos de consumo, de vivienda, microcrédito individual y micro apoyo, sino que también ofertan soporte al sector productivo; de ahí la importancia de la participación de esos agentes.

Fue también determinado la participación universitaria; en este caso profesores y estudiantes a participar por carreras. Se conformaron los equipos con la participación de estudiantes de las carreras de Ingeniería Agrícola y de Administración de Empresas.

En esta comunidad rural, se mantiene una tendencia de migración visible del área rural a la ciudad en busca de mayores oportunidades; ya que, muchos de los microproductores han perdido medios de producción y se les ha cerrado la posibilidad de acceso a los créditos, todo lo cual ha hecho que los rendimientos no sean relevantes ni suficientes.

Al igual que el diagnóstico efectuado en los inicios de la investigación, se pudo observar, mediante los planes estratégicos de desarrollo del área de vinculación con la comunidad, que la universidad aún no había logrado un protagonismo visible a través de sus procesos para lograr revertir la situación de la comunidad rural; no obstante, a partir de la investigación que se desarrolló, comenzaron a organizarse proyectos para la solución de la problemática de la comunidad.

\section{Conclusiones}

La extensión debe desarrollarse desde un modelo que propugne la responsabilidad social universitaria, para que puedan enrumbarse los microproductores por el camino del desarrollo.

El diagnóstico efectuado para valorar el estado en que se encontraba la problemática en el cantón Bolívar, reveló que era insuficiente la preparación que se le brindaba a

los microproductores, hecho por el cual, los indicadores de emigración, pobreza y marginalidad estaban en ascenso.

La Estrategia Educativa de extensión universitaria para el desarrollo de la cultura económica en el microproductor agrícola fue concebida sobre la base de las demandas de la universidad para el presente siglo, los procedimientos y principios de la educación popular y la gestión de la extensión como propósito transformador. 


\section{Referencias bibliográficas}

Acconcia, M. L., Alvarez, M. V., Michniuk, N., Villanueva, M. L., Ibañez, V., Ali, S. V., \& Henríquez, G. (2017). La extensión universitaria como espacio dialógico de construcción y problematización de saberes y prácticas.

Achugar, H. (2000). Desafíos económico-culturales de América Latina (cultura" tradicional" e industrias culturales). Capital social y cultura: claves estratégicas para el desarrollo México DF: BID-FCE.

Antillón, N. R. (2002). ¿Cómo lo hacemos?... para construir conocimiento a través de la sistematización de la práctica social. IMDEC, México.

Araujo, L. (2009). Para una socioeconomía de la pequeña empresa. Estado de la cuestión. Descripción estadística. Caracas: UCAB. Tesis doctoral.

Aristizábal, A. B. (2006). La Educación para el Desarrollo orientada al Desarrollo Humano. In Construir la ciudadanía global desde la universidad: propuestas pedagógicas para la introducción de la educación para el desarrollo en las enseñanzas científico-técnicas (pp. 39-52). Intermón Oxfam.

Barnett, R. (2002). Claves para entender la universidad: en una era de súper complejidad. Ediciones Pomares.

Barretto Ghione, H. (2007). Formación profesional en el diálogo social. Montevideo: Cinterfor/OIT.

Boni, A. (2006). La Educación para el desarrollo orientada al desarrollo humano. En: Boni, A. \&amp; Pérez Foguet, A. (Eds.). La Construcción de la Ciudadanía Global. Barcelona: Colección Informes. Ed. Intermón-Oxfam

BID (2009). La Microempresa en Ecuador. Perspectivas, Desafíos y Lineamientos. Ecuador: McGraw-Hill. Banco Interamericano de Desarrollo.

Carvajal, E., Auerbach, P., Vivanco, F., \& Guerrero, M. (2006). La Microempresa en Ecuador: perspectivas, desafíos y lineamientos de apoyo. Ecuador: Banco internacional de Desarrollo Representación Ecuador.

Cabrera, O. R. (2017). www.nodo50.org/cubasigloxxi/owww.filosofia.cu. Obtenido de Filosofia.cu.: http://www. nodo50.org/cubasigloxxi/owww.filosofia.cu

Castells, M. (2004). http//www.fbg.ub.es. Obtenido de http://www.fbg.ub.es.: http//www.fbg.ub.es

Constitución de la República del Ecuador (2008). Constitución. Montecristi: Corporación de Estudio.

De La Torre. (2001). La universidad que necesitamos. México: Juan Pablos Editor, S.A.

De Sousa Santos, B. (2010). La universidad del siglo XXI. Para una reforma democrática y emancipatoria de la universidad. Ediciones Trilce. 
Diccionario Etimológico (2019). Concepto de Estartegia. Diccionario de Etimologia, https://definicion.de/estrategial

FAO-FIDA-OIT (2010). Investing in Skills for Socio-Economic Empowerment of Rural. Rome: Women, Policy Brief.

Freire, P. (1975): Pedagogía del oprimido, México: Siglo XXI Editores.

Freire, P. (1976): La educación como práctica de la libertad, México: Siglo XXI Editores.

Garzón, M.F. (2017). Modelo de educación posgraduada para el desarrollo regional. Revista Transformación, 13 (2), 184-191.nt Tomadode:http://revistas.reduc.edu.cu/index.php/transformacion/article/view/1425/pdf

Garzozi Pincay, R., Messina Scolaro, M., Moncada Marino, C., Ochoa Luna, J., Pérez, G. I., \& Zambrano Segura, R. (2014). Planes de negocios para emprendedores.

Geerlings, J. (1999). Design of responsive vocational education and traning.

Gómez, M. V. (2001). Educación a distancia y cátedras libres: reflexionando sobre emergentes en el contexto de la educación latinoamericana. CLACSO.

González, R. (2018). Estrategia Educativa de Extensión Universitaria para la cultura económica de los microproductores agrícolas. Camagüey: Tesis doctoral. Universidad de Camagüey Ignacio Agramonte Loynaz.

Jara (2010). Educación popular y cambio social en América Latina. USA: Community Development Journal. Tomado de: http://cdj.oxfordjournals.org/content/suppl/2010/06/28/bsq022.DC1/bsq022 supp.pdf

Núñez, D. (2014). Gestión de la dinámica formativa cultural universitaria, caso Universidad Estatal de Bolívar. Santiago de Cuba: Tesis doctoral.

Peña, G. y Guerra, B. (1990). La conciencia económica en la conciencia social. La Habana: Editora Política.

Registro Oficial 449. (2008). La Constitución. Obtenido de: https://www.oas.org/juridico/pdfs/mesicic4 ecu const.pdf

Robalino, M. (2005). ¿Actor o protagonista? Dilemas y responsabilidades sociales de la profesión docente. Revista Prelac, 1 (Proyecto Regional de Educación para América Latina y el Caribe).

Russo, H. (2000). La educación ¿sigue siendo estratégica para la sociedad? CLACSO.

Sabalain, C. (2011). El concepto de rural en los países de la región. Dirven M., R. Echeverri, C. Sabalain, A. Rodríguez, D. Candia Baeza, C. Peña y S. Faiguenbaum. Hacia una nueva definición de "rural" con fines estadísticos en América Latina, 3, 21-29. 
Sojo, A., Uthoff, A., \& CEPAL, N. (2007). Cohesión social en América Latina y el Caribe: una revisión perentoria de algunas de sus dimensiones.

UNESCO. (2009). Conferencia Mundial de Educación Superior \&quot; Las Nuevas Dinámicas de la Educación Superior y de la investigación para el cambio social y el desarrollo\&quot., París, Francia.

Van de Velde, H. (2002). La sistematización de experiencias educativas: un espacio para la reflexión crítica y la transformación de la práctica. Managua: IDE-UCA. 
Copyright (c) 2020 Rosa González Zambrano, Benjamín Wilson León Valle, René Garzozi Pincay, Lourdes Crespo Zafra y Jorge Saavedra Palma

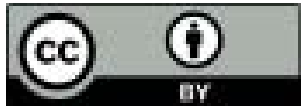

Este texto está protegido bajo una licencia internacional Creative Commons 4.0.

Usted es libre para Compartir - copiar y redistribuir el material en cualquier medio o formato - y Adaptar el documento - remezclar, transformar y crear a partir del material-para cualquier propósito, incluso para fines comerciales, siempre que cumpla las condiciones de Atribución. Usted debe dar crédito a la obra original de manera adecuada, proporcionar un enlace a la licencia, e indicar si se han realizado cambios. Puede hacerlo en cualquier forma razonable, pero no de forma tal que sugiera que tiene el apoyo del licenciante o lo recibe por el uso que hace de la obra.

\section{$\underline{\text { Resumen de licencia - Texto completo de la licencia }}$}

